\title{
Coronal current sheet signatures during the 17 May 2002 CME-flare
}

\author{
H. Aurass ${ }^{1}$, F. Landini ${ }^{2}$, and G. Poletto ${ }^{3}$ \\ 1 Astrophysikalisches Institut Potsdam (AIP), 14482 Potsdam, Germany \\ e-mail: haurass@aip.de \\ 2 University of Firenze, Astronomy and Space Science Deptm., 50125 Firenze, Italy \\ 3 INAF - Osservatorio Astrofisico di Arcetri, 50125 Firenze, Italy
}

Received 30 March 2009 / Accepted 17 August 2009

\begin{abstract}
Context. The relation between current sheets (CSs) associated with flares, revealed by characteristic radio signatures, and current sheets associated with coronal mass ejections (CMEs), detected in coronal ultraviolet (UV) and white light data, has not been analyzed, yet.

Aims. We aim at establishing the relationship between CSs associated with a limb flare and CSs associated with the CME that apparently develops after the flare. We use a unique data set, acquired on May 17, 2002, which includes radio and extreme ultraviolet (XUV) observations.

Methods. Spectral radio diagnostics, UV spectroscopic techniques, white light coronograph imaging, and (partly) radio imaging are used to illustrate the relation between the CSs and to infer the physical parameters of the radially aligned features that develop in the aftermath of the CME.

Results. During the flare, several phenomena are interpreted in accordance with earlier work and with reference to the common eruptive flare scenario as evidence of flare CSs in the low corona. These are drifting pulsating structures in dynamic radio spectra, an erupting filament, expanding coronal loops morphologically recalling the later white light CME, and associated with earlier reported hard X-ray source sites. In the aftermath of the CME, UV spectra allowed us to estimate the CS temperature and density, over the 1.5-2.1 $R_{\odot}$ interval of heliocentric altitudes. The UV detected CS, however, appears to be only one of many current sheets that exist underneath the erupting flux rope. A type II burst following the CME radio continuum in time at lower frequencies is considered as the radio signature of a coronal shock excited at the flank of the CME.

Conclusions. The results show that we can build an overall scenario where the CME is interpreted in terms of an erupting arcade crossing the limb of the Sun and connected to underlying structures via multiple CSs. Eventually, the observed limb flare seems to be a consequence of the ongoing CME.
\end{abstract}

Key words. Sun: magnetic fields - Sun: coronal mass ejections - Sun: flares - Sun: radio radiation - Sun: UV radiation

\section{Introduction}

During coronal energy release phenomena such as confined or eruptive flares, eruptive prominences, and coronal mass ejections (CMEs), structural changes of the coronal magnetic field take place. It is now (at least after the $Y O H K O H$ mission, Tsuneta et al. 1992) generally accepted that magnetic reconnection (Biskamp 2000) is a key process in the solar corona. When two oppositely directed magnetic flux elements approach each other to more than some ion cyclotron radii ${ }^{1}$ their connectivity with the photosphere can be changed, a "field diffusion" region is formed. In 2D geometry, a current flows in the diffusion region perpendicular to the plane of the field lines. In a real case, the diffusion region may have a large extent in that direction (as a thin, possibly curved surface) justifying the term "current sheet" (see e.g. Somov 2000).

Independently of how a coronal CS can be directly "seen", its presence during coronal energy release is highly probable. The CS is a central part of cartoons, theoretical and numerical models of prominence eruptions (e.g. Anzer \& Pneuman 1982), flares (e.g. Martens \& Kuin 1989; Masuda et al. 1994; Priest \& Forbes 1999), and CMEs (e.g. Hundhausen 1998; Saint-Hilaire et al. 2009) as a site for energy release.

\footnotetext{
1 Some meters in the corona.
}

Flares and CMEs proceed on different time- and spatial scales, and can occur in association, but also be independent of each other. Zhang et al. (2004) conclude that "CMEs and flares are physically integrated phenomena ... both are the results of more fundamental processes".

Models of flares and CMEs involve a CS as an essential part. How do these "flare-CSs" and "CME-CSs" fit together? How are they associated in space and time? Is it the same situation as with the erupting active region filament or the erupting prominence that can be (sometimes) later seen in white light as a bright part of the CME, or is the situation completely different?

We search for an answer using an example, a near-limb flare and an associated CME, and analyze several indirect indicators for the presence of CSs. These are characteristic radiative signatures in radio and HXR excited by electrons accelerated in the flare-CS, on the one hand, and excessively heated regions (observable by UV spectroscopy) of more or less radial extent trailing the white light CME above the coronograph occulter, on the other.

The problem we mean to face implies using different diagnostics at different times and applying them at data originating in different coronal sites. We need to fit observations that have been taken sequentially and build a unique temporal and spatial scenario. In an attempt to closely reproduce what is occurring during our event we first examine radio spectral and X-ray flux 

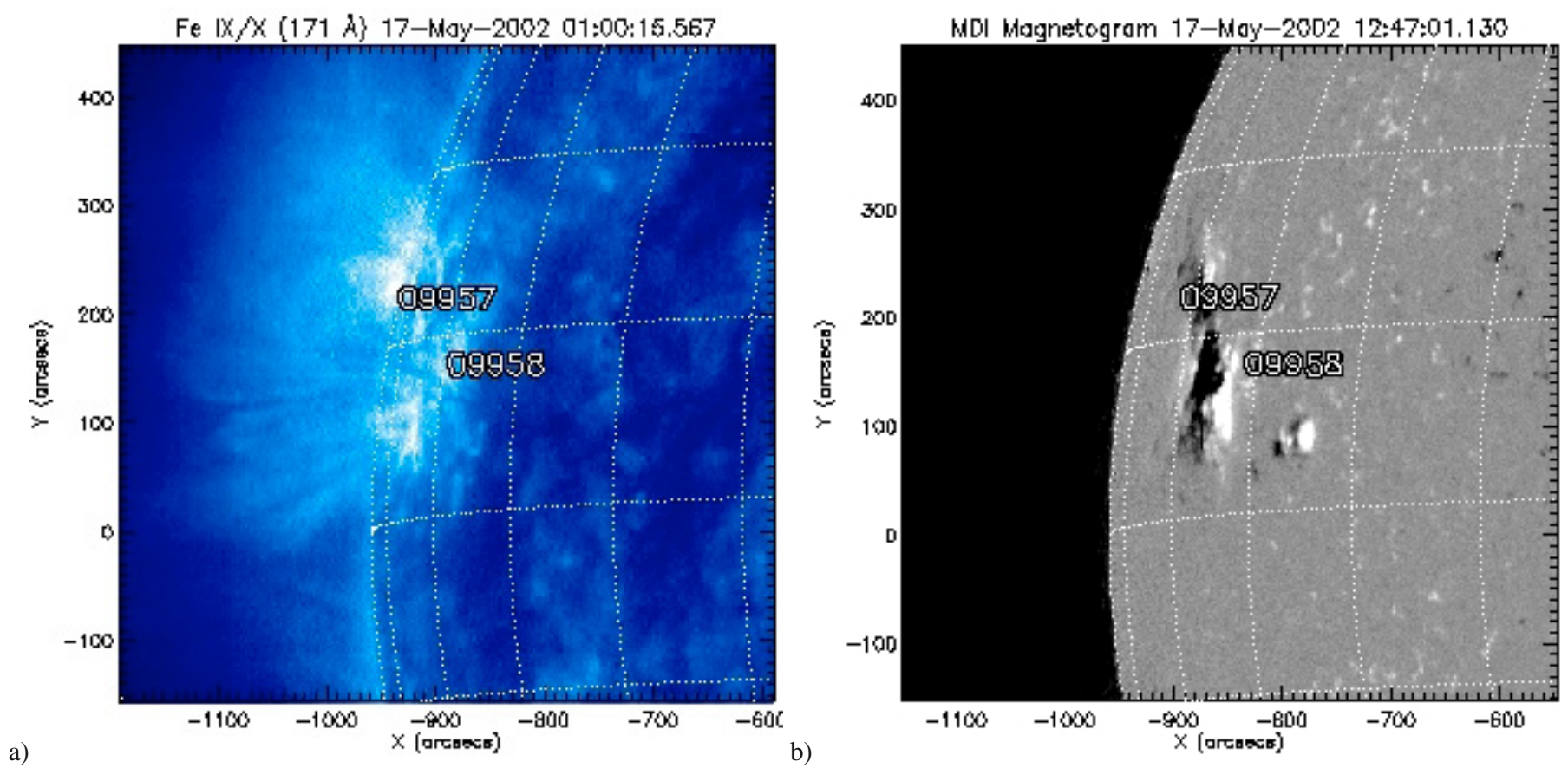

Fig. 1. SOHO-EIT $171 \AA$ A image a) and SOHO-MDI magnetogram b) on May 17, 2002 that show the context within which the CME analyzed in this paper took place. North at top, East to the left. ARs 9957 and 9958 are denoted in both panels.

records and try to establish a temporal sequence with the help of white light images, while we resort to a potential field extrapolation to organize data in a spatial frame. Before describing the event, we give some basic information about the data we used in different spectral ranges.

Decimetric radio bursts are a characteristic impulsive flare phase signature, correlated with the flare HXR emission and excited at the lowest levels of the solar corona (Dulk et al. 1985). Benz (1986), Aurass \& Kliem (1992), Karlický et al. (2002), and Karlický (2004) were the first who noticed that characteristic spectral fine structures in the dynamic spectrograms of decimeter emission are indicative of the dynamic behavior of the primary energy release site. Hori (1998) has confirmed that idea-she has shown that decimetric drifting pulsating structures (DPS) are associated with rising blobs of matter seen in soft X-rays. Following Karlický (2004) we consider such fine structures as an indicator of the very existence of a CS in the early stage of the flare, when an associated CME is not yet visible in coronograph images. Kliem et al. (2001) and Bárta \& Karlický (2007) have stressed the significance of associating DPSs with the dynamic evolution of flare CSs by MHD-model calculations, and combined MHD-model and particle-in-cell-code simulations, respectively.

Later on the event time scale, the CME rises over the LASCO coronograph occulter. Its morphology is well described by the terms leading loops, cavity and core (Hundhausen 1998). Underneath that expanding structure, a current sheet is predicted (see, e.g. Hundhausen 1998; Priest \& Forbes 1999), and sometimes thin and twisted plasma structures are reported to connect the CME with low-corona features covered by the coronograph occulter. A breakthrough observation by the SOHO-UVC spectrograph revealed in such a thin radial structure between the CME and the occulter high emission in the [Fe XVIII] $\lambda 974 \AA$ line, pointing to the presence of temperatures $\geq 6 \times 10^{6} \mathrm{~K}$ over spatial scales on the order of $5 \times 10^{4}$ to $5 \times 10^{5} \mathrm{~km}$ (Ciaravella et al. 2002). These hot features have been found in many CMEs (see, e.g. Ko et al. 2003; Bemporad et al. 2006; Ciaravella \& Raymond 2008) and their identification with CSs has been further supported by observations of plasma flowing along the radial structures, identified as reconnection outflows (see, e.g. Ko et al. 2003), and of blobs, interpreted in terms of the dynamic behavior of a CS (see e.g. Lin et al. 2005).

In the literature the UVCS features are referred to as CSs although this term is not consistent with its use in analyzing lower corona data as e.g. radio observations. There is no doubt that the flare CS can be observed indirectly, only. Estimates (Vršnak et al. 2009) demonstrate that coronograph images can also include the slow-mode shock range above and below the diffusion region ${ }^{2}$ so that the derived dimensions in heights above the LASCO-C2 occulter are not unreasonable. In principle, so the coronograph images give CS evidence in the same manner as the limb-flare X-ray images of Masuda et al. (1994) which was also considered as a breakthrough observation: the X-ray images do not show the diffusion region but the surrounding heated corona and a part of the slow-mode shocks with the characteristic inverted Y-shaped hot matter. It is beyond the purpose of the present work to continue this discussion. We will, for the moment, accept both CS indicators based on the quoted previous work.

\section{May 17, 2002-data analysis}

\subsection{Overview}

The event we analyze started at about 7:30 UT, on May 17, 2002, at the East limb of the Sun, above the active region complex which includes AR 9957 and 9958, located at, respectively, N06, E53 and N03, E45 and followed, at a slightly higher latitude, by AR 9960 (see Fig. 1). AR 9960 appears at the limb of the Sun, on May 18 , at a latitude of $\approx 18^{\circ}$ and in the southern hemisphere, AR 9955 and AR 9954 are at, respectively, S14, E 41, and S22,

${ }^{2}$ We refer to the same paper also for the explanation of terms. 
Table 1. May 17, 2002-time line.

\begin{tabular}{|c|c|c|c|}
\hline Wave band & Figure & $\begin{array}{l}\text { Instrument/ } \\
\text { observatory }\end{array}$ & $\begin{array}{l}\text { Description } \\
\text { (times in UT, speeds in } \mathbf{k m ~ s}^{-1} \text {, angles, colatitude, degrees, } \\
\text { counterclockwise from North Pole) }\end{array}$ \\
\hline WL & & LASCO-C2 & $\begin{array}{l}\mathrm{CME} \text { at } 01: 50 \text { E } 90^{\circ} v=\mathbf{3 7 0} \text {, width }=38^{\circ} \\
04: 26 \mathrm{E} 91^{\circ} v=\mathbf{1 2 3} \text {, width }=10^{\circ}\end{array}$ \\
\hline X-rays & & GOES & 05:16-05:28 (AR 9957) \\
\hline H-alpha & & SVTO & 05:20-05:34 (AR 9957) \\
\hline X-rays & 3 & GOES & 07:27-07:54->08:40 M1 \\
\hline UVB & & CELIAS & 07:30 onwards: SEM flux increase \\
\hline hard X-rays & & RHESSI $^{10}$ & 07:32-07:36 double source, motion, $07: 37$ single source at $\mathrm{E} 76^{\circ}$ \\
\hline Radio & & $\mathrm{AOO}$ & 07:32- >08:12 $3 \mathrm{GHz}$ complex dm burst, main max. 08:06 \\
\hline & 3 & AOO, AIP & 07:37-07:59 1800-350 MHz drifting pulsating structures \\
\hline EUV & 1 & EIT & 07:36 first rising loop image $195 \AA$ at $\mathrm{E} 76^{\circ}$ \\
\hline Radio & 3 & AIP, WIND & $07: 41-07: 4680-1 \mathrm{MHz}$ IIIG, changing $f_{\text {start }}$ \\
\hline EUV & 1 & EIT & $07: 48$ second image of the loop, $v_{\text {rise }}=\mathbf{1 3 5}$ \\
\hline \multirow[t]{4}{*}{ Radio } & 3 & AIP, WIND & 07:52-07:59 IIIG, 07:59 sudden end of m-Dm IIIG KA \\
\hline & & AIP & 07:56-07:58 faint narrowband pattern in $360-420 \mathrm{MHz}$ \\
\hline & 3 & AIP & $\approx 08: 00-08: 10$ CME continuum \\
\hline & & $\mathrm{AOO}$ & 08:06 main $3 \mathrm{GHz}$ pulse \\
\hline \multirow[t]{2}{*}{ WL } & 2 & LASCO-C2 & $\mathrm{CME}$ 08:06 E $61^{\circ} v=\mathbf{6 6 5}$, width $=92^{\circ} \mathrm{GO}$ \\
\hline & 2 & & 08:06 E $61^{\circ} v=347$, width $=95^{\circ} \mathrm{OLZ}$ \\
\hline \multirow[t]{2}{*}{ Radio } & 3 & AIP & 08:14-08:23 II 55-40 MHz \\
\hline & 4 & $\mathrm{NRH}^{11}$ & radio loop $\mathrm{E} 76^{\circ} h \approx 70 \mathrm{Mm}$ at $327 \mathrm{MHz}$ \\
\hline \multirow[t]{2}{*}{ Optical } & & WROC & $<08: 27-12: 24$ Erupt. prominence at limb N15E90 \\
\hline & 4 & AIP, NRH & 09:11:30-09:12:30 IIIG 500-260 MHz E $110^{\circ}$ \\
\hline WL & & LASCO-C3 & CME 08:42 UT (cf. Footnote 1, Sect. 2.2) \\
\hline \multirow[t]{2}{*}{ EUV } & 5 & UVCS & 08:28-9:58 slitpos. E $90^{\circ}$ covering postCME CS \\
\hline & 5 & & from 2.1-1.5 $R_{\odot} 9: 13-9: 58$ OVI reduced, SiXII enhanced \\
\hline X-rays & & GOES & 12:05 E 82 \\
\hline H-alpha & & SVTO & $16: 11$ E 83 \\
\hline X-rays & & GOES & 23:06 E 63 (AR 9957) \\
\hline H-alpha & & HOLL & 23:07 E 63 (AR 9957) \\
\hline WL & & LASCO-C2 & CME 13:50 E 89 \\
\hline WL & & LASCO-C2 & CME 16:26 E 95 \\
\hline IP shock & & CELIAS & May 20, 02:56 UT \\
\hline
\end{tabular}

${ }^{10}$ Images to $40 \mathrm{keV}$ in Karlický et al. (2004); end of RHESSI (Ramaty High Energy Solar Spectroscopic Imager) obs. at 07:39 UT; ${ }^{11}$ start of obs. at 08:27 UT.

Abbreviations: UVB: UV broadband, GO: /cdaw.gsfc.nasa.gov/CME_list, OLZ: /spaceweather.gmu.edu/seeds, KA: /lep694.gsfc . nasa.gov/waves, AIP: Astrophysikalisches Institut Potsdam; AOO: Astronomical Observatory Ondřejov; NRH: Nançay Radio Heliograph; SVTO: San Vito, HOLL: Holloman.

E41. This complex has been the site of frequent activity, both before and after 7:30 UT. In Table 1 we give a list of the relevant observations that have been done at the time of our event. We also list the $\mathrm{H} \alpha$ - and/or GOES-detected flares, and CMEs that occurred prior and after our event, on the same day and, most likely, from the same AR complex: when the AR number appears in the Solar Geophysical Data, its number is given in the Table. Data have been arranged sequentially in time.

The EIT $195 \AA$ images show diffuse emission, centered at a latitude of $\approx 12-14^{\circ}\left(\mathrm{PA}=76-78^{\circ}, \mathrm{PA}=\right.$ Position Angle (degrees), measured counterclockwise from solar North), above this AR complex. Figure 2 gives a sequence of EIT $195 \AA$ running difference images that cover the time interval from 07:36 to $08: 24$ UT and illustrate the initial phase of our event: bright loops are seen to form and rise upwards in agreement with reports of an eruptive prominence at PA $=75^{\circ}$ at $<08: 27$ UT. Evidence for a progressive rising and weakening of these structures comes from later images.

Indeed, looking at the $\mathrm{C} 2$ images in Fig. 2b, we see a CME first appearing above the occulter at $\approx 8: 06$ UT. The CME is well seen in these images (and in, e.g., the wavelet enhanced images in Fig. 4): however, it is not easy to understand whether we see two CMEs that develop successively - the northern one originating slightly after the first one - or whether we look at a single event, which spans over a large latitude interval, partially extending behind the limb. If this is the case, and the event spans over a large longitude interval as well, loops which originate from behind the limb, when projected onto the plane of the sky, culminate closer to the limb. In the $\mathrm{C} 3$ coronagraph our event is first imaged around 08:42 $\mathrm{UT}^{3}$ confirming that the eruptive events imaged in the lower corona reach the outer coronal levels.

The EIT and LASCO data set the stage for the following discussion of the event in radio (AIP and AOO) and SOHO-UVCS observations (see Table 1).

\subsection{Radio and X-ray observations}

In this work we use the dynamic radio spectra of AIP and the corresponding time intervals of AOO (Karlický et al. 2004) shown

\footnotetext{
3 The CME speed should be almost twice as large as appears in the CME catalog!
} 

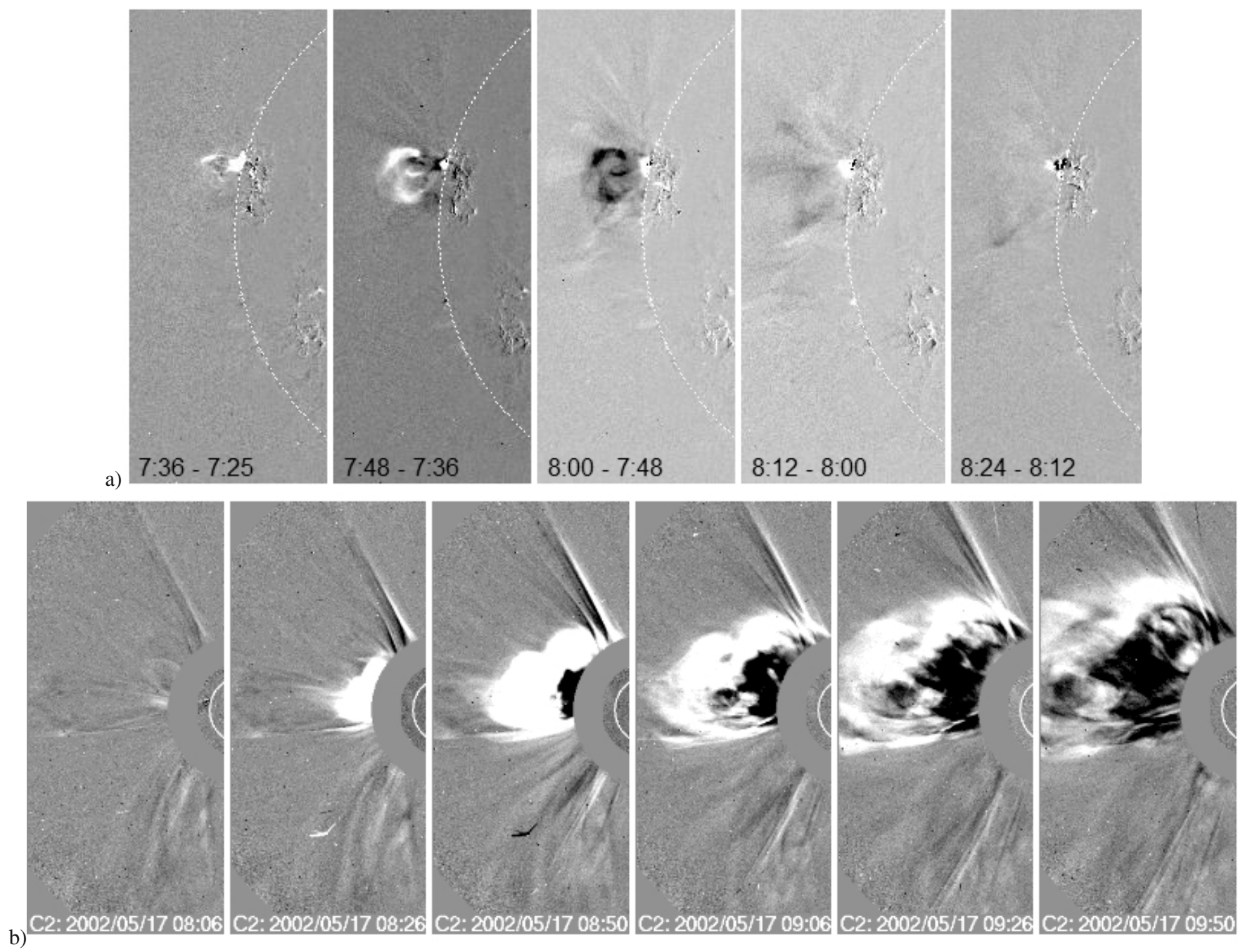

Fig. 2. SOHO data of May 17, 2002. North is top, East to the left. The limb of the sun is the white arch. a) EIT $195 \AA$ running difference images from 7:36 to 8:24 UT. Between 7:32 and 7:37 UT, RHESSI recorded 30-40 keV sources at PA76 ${ }^{\circ}$ E (Karlický et al. 2004, and Table 1). A meter wave radio continuum is observed with a $327 \mathrm{MHz}$ centroid height of $\approx 70 \mathrm{Mm}$ at the same PA later on (after the NRH morning at 8:27 UT), see Fig. 4. b) LASCO-C2 running difference images of the CME first imaged at 8:06 UT. The C2 occulter is the grey shaded area. For wavelet enhanced LASCO-C2 images see Fig. 4. At first glance, the East-limb CME consists of a southern (earlier starting) and a northern (later occuring) part.

in Fig. 3, and the NRH images available after 08:27 UT. The radio burst emission is of nonthermal origin-it is driven by the presence of nonthermal electrons in the coronal plasma.

Taking into account that we aim at establishing the spatial/temporal relationship between flare CSs and the later observations of CSs below a CME, we summarize the information from radio data as follows:

- The continous presence of nonthermal electrons in the corona over a large time interval, at least from 07:3608:23 UT. GOES X-ray flux records (Fig. 3) and RHESSI HXR observations (Karlický et al. 2004) extend this time interval to 07:29- $\gg 08: 20$ UT. NRH imaging data show at the PA of the former HXR source site a long-duration postflare continuum source centroid, and - more to the South-a type III burst group source at 09:10-09:12 UT.

- In the spectrum, a low-frequency cut-off drifts toward lower frequencies, conservatively at least between 400 and $40 \mathrm{MHz}$, and between 07:48 and 08:23 UT. At still lower frequencies, type III bursts occur only sometimes. In 08:1508:23 UT, $55-\approx 40 \mathrm{MHz}$, the radio record of the event terminates with a single type II burst lane.
- The dm effects between $2 \mathrm{GHz}$ and $800 \mathrm{MHz}$ are classified as drifting pulsating structures (DPS, Karlický et al. 2004, and personal communication) pointing to the presence of dynamic processes in flare CSs (Karlický 2004).

- The correlation between radio spectral data and X-ray data is changing over time. This is not unusual but has, nevertheless, recently been further discussed in an independent analysis (Benz et al. 2007). We note the following relation:

- The event is radio-quiet in the $\mathrm{dm}-\mathrm{m}$ range in the early impulsive phase (07:29-07:38 UT);

- After 07:38 UT, and during the decay of X-ray emission (07:40-07:46 UT) there is DPS and type III group emission $(170-<40 \mathrm{MHz})$;

- In a next energy release phase (07:46-07:59 UT) we find DPS emission between $>2 \mathrm{GHz}$ and $350 \mathrm{MHz}$ with spectral fine structures in 07:57-07:59 UT, 800-600 MHz. It is interesting that the type III bursts (previous item) extend down to very low frequencies (see the 14$1 \mathrm{MHz}$ data from WIND, not shown here) exactly until 07:59 UT. Later occuring type III bursts (08:10:30 and 09:11:30 UT) do not reach the WIND frequency range; 


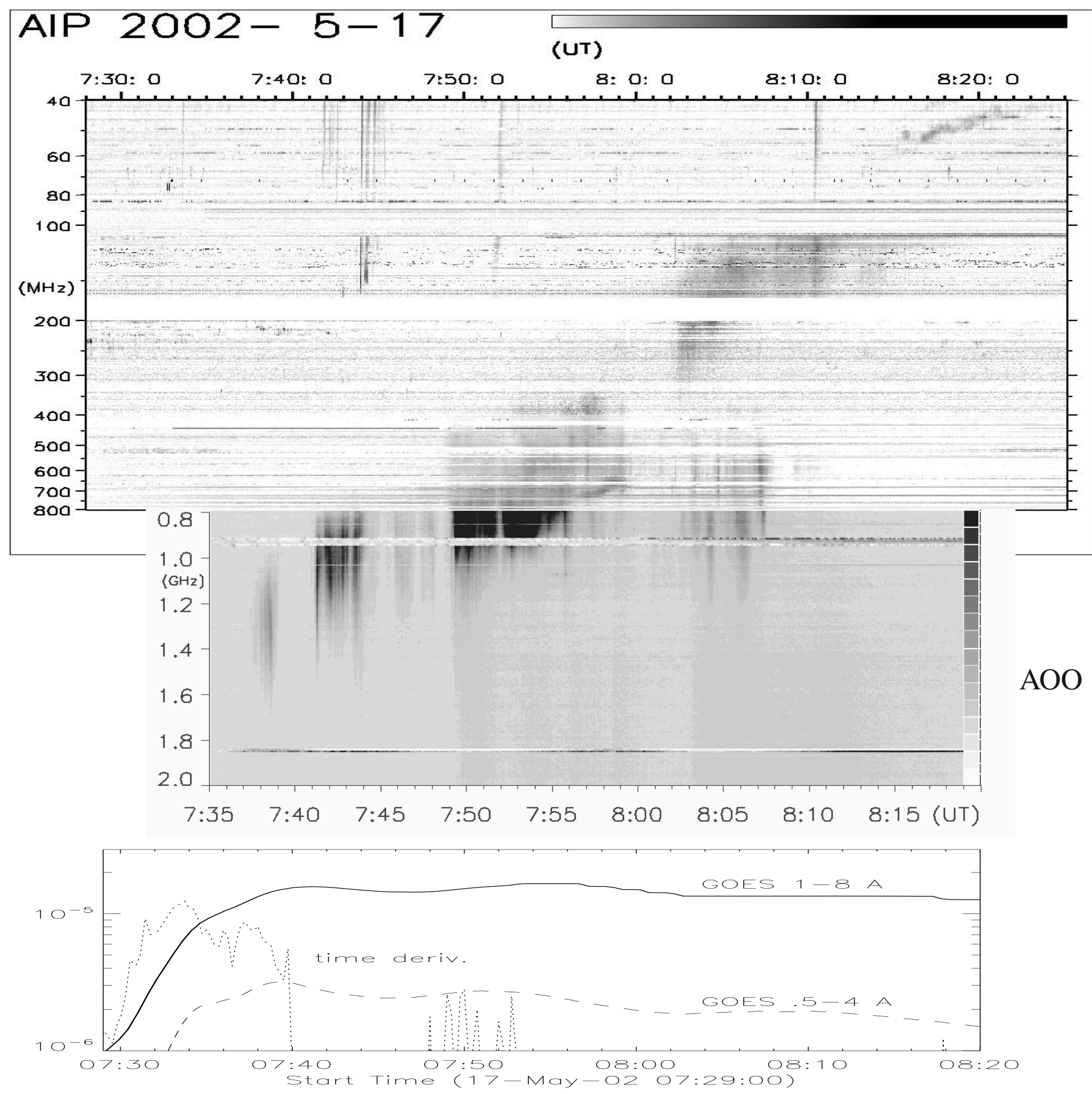

Fig. 3. Dynamic radio spectra of AIP (top) and AOO (middle, after Karlický et al. 2004, and http://www.asu.cas.cz/\%7Eradio/). Lines parallel to the time axis are disturbances, time-parallel lanes are frequency bands completely covered by strong radio and TV signals. The AIP spectrum is the difference with respect to a first spectral scan in the plot. Flux data (grey shaded) are in arb. units and logarithmic. Bottom: GOES flux $\left(\mathrm{W} \mathrm{m}^{-2}\right)$ and 1-8 $\AA$ (positive valued) time derivative in arb. units.

- After a gap in the spectrum (07:59-08:02 UT) there is only faint DPS emission remaining $(1.2 \mathrm{GHz}-500 \mathrm{MHz})$, and a smooth "CME-continuum" (in the sense of Aurass et al. 1999; Bastian et al. 2001) occurs in >300$\approx 100 \mathrm{MHz}(08: 02-\approx 08: 14 \mathrm{UT})$. A single type III burst is superposed at 08:10:30 UT. All this is accompanied by a slow-down of the decay or a faint re-enhancement of the X-ray flux.

The X-ray data (GOES) are used for reference with the radio data in the present context. The HXR imaging (RHESSI) is available until 07:39 UT. A HXR source is seen in 6-15 keV as discussed in detail by Karlický et al. (2004). The source is located at the same place where the rising EIT loop system appears (see Fig. 2) in the impulsive flare phase, where the first CME signature was imaged by LASCO-C2, and where NRH images show a postflare continuum after 08:27 UT.

We consider the radio observations as a link connecting $\mathrm{X}$-ray flare imaging, on the one hand, and LASCO-CME imaging, on the other hand. The radio spectral diagnostics informs about the presence of dynamic flare CSs (DPS), and about the 
Table 2. UVCS slit positions and time intervals used in our analysis.

\begin{tabular}{cc}
\hline \hline Heliocentric height & Time interval \\
\hline$\left(R_{\odot}\right)$ & $($ hh:mm-hh:mm $)$ \\
2.6 & $08: 46-09: 13$ \\
2.1 & $09: 13-09: 30$ \\
1.9 & $09: 30-09: 43$ \\
1.7 & $09: 43-09: 53$ \\
1.5 & $09: 53-09: 58$ \\
\hline
\end{tabular}

presence and motion of the CME in the lowest coronal heights (CME-continuum). Eventually, sudden changes or interruptions of ongoing radio emission ${ }^{4}$, point to large-scale dynamics in the corona associated with the CME motion (comp. Khan \& Aurass 2006).

\subsection{Ultraviolet Coronagraph Spectrometer (UVCS) observations}

At the time of the event, on May 17, 2002, UVCS was operating in the synoptic mode: that is, the UVCS slit, set normal to the solar radius, at different altitudes, moves sequentially all around the Sun, in steps of $45^{\circ}$. From 8:28 to 9:58 UT, the slit was normal to the equatorial radius, in the eastern hemisphere, and covered a range of altitudes from 1.5 to $3.1 R_{\odot}$. UVCS acquired spectra in three wavelength intervals, 971.65 $\leq \lambda \leq 985.56$, $997.97 \leq \lambda \leq 1000.75,1000.95 \leq \lambda \leq 1043.2$ with a spatial binning of 21 arcsec/pixel and a spectral binning of $0.27 \AA$ per pixel in the first spectral interval and of $0.18 \AA$ per pixel in the others. The exposure time was $120 \mathrm{~s}$. The total observing time- $5 \mathrm{~min}$ at $1.5 R_{\odot}$-increased to longer durations when UVCS takes data at higher altitudes. Within the spectral range covered by the spectrograph, the strongest spectral lines are the $\mathrm{H} \mathrm{Ly-} \alpha$, the OVI $1032 \AA$ and the SiXII $499 \AA$ line, observed in the second order. Notice that the slit is first set at the highest altitude $\left(3.1 R_{\odot}\right.$ for this data set) and then moves down. Hence the lower coronal plasma is observed at the end of the observations.

The data have been wavelength and flux calibrated. We first integrated over the line profile, to obtain the total intensity of the observed lines. However, we had to integrate over 5 spatial pixels (thus decreasing the spatial resolution) and sum, at each slit position, over the whole exposure time to obtain a statistically significant signal. We ended up with six spectra, acquired at $R=1.5$, $1.7,1.9,2.1,2.55,3.1 \times R_{\odot}$, each corresponding to a different time interval. Table 2 summarizes the times and slit positions. The two spectra acquired at 3.1 and $2.55 R_{\odot}$ were discarded, because line intensities decrease with altitude and in spite of the longer exposures the SixII line became too weak to be statistically significant. Figure 4 has shown the slit positions at 8:50 and 09:26 UT superposed onto LASCO-C2 contrast-enhanced images.

We aim at finding evidence for the presence of a CS at the heliocentric heights sampled by UVCS. As described in Sect. 1, CSs have been identified via the emission in the $974 \AA$ line of the [FeXVIII] ion. Unfortunately, this line is not included in synoptic UVCS spectra and we have to adopt alternative techniques. In the past, in a comparable situation, e.g. Lin et al. (2005) resorted to an analysis of UVCS HI Ly- $\alpha$ data. Aligned with the LASCO white light $\mathrm{CS}^{5}$ Lin et al. (2005) noticed a spatial interval over which the UVCS Ly- $\alpha$ line had a decreased brightness, as

\footnotetext{
${ }^{4}$ Here: $\mathrm{dm}$ radiation depletion between 07:59 and 08:02 UT, and type III burst total bandwidth reduction thereafter.

${ }^{5}$ Identified by the bright "stem" located below the CME core.
}

expected if the region is filled with hot plasma. Also, the width of the interval with reduced Ly- $\alpha$ intensity decreased in time, as expected in case of reconnection inflows.

The faint Ly- $\alpha$, the temporal behavior of the spatial interval where this occurred, and the spatial correlation with the LASCO bright feature, were deemed by the authors to point to the presence of a CS. In the present work, we resort to an analogous procedure to identify signatures of a CS in UVCS data.

Figure 5 gives the total line intensity profiles, along the UVCS slit, of SiXII and OVI lines at $1.9 R_{\odot}$. The coronograph occulter and the disk of the sun (white) are shown at right. A radius is plotted through $\mathrm{PA}=83^{\circ}$ for reference (see Fig. 4). The $x$-axis of the two plots are set normal to the equatorial radius, to reproduce the UVCS slit position and cover the $\approx 2000$ arcsec over which UVCS took data. The $x$-axis of the OVI plot is set at an heliocentric distance of 1.9 solar radii, in agreement with the position of the UVCS slit. Each unit on this axis is $100 \operatorname{arcsec}$. The $y$-axis gives the total counts observed in the lines.

Figure 5 shows that:

- a OVI line minimum appears at a position, along the UVCS slit, corresponding to PA $83^{\circ}$ and;

- at this position the SiXII line peaks, as expected in a region of enhanced temperature.

To rule out a fortuitous occurrence of the OVI/SiXII intensity anticorrelation, we checked for its occurrence over all the heliocentric distances where significant line emission had been observed. It turns out that the anticorrelation occurs through all levels, from 1.5 to $2.1 R_{\odot}$, sampled by UVCS over a time interval of $\approx 45 \mathrm{~min}$.

LASCO C2 wavelet enhanced images show the CME rising at increasingly larger heliocentric distances. Under the rising core, a bright "stem" is recognizable, at the position where, according to the Lin and Forbes (2000) model, we expect a CS. Its PA coincides with the position angle where the OVI/SiXII line intensities minimizes/maximizes.

The examined images and spectra lead us to conclude that the bright white light feature corresponds, at the lower levels sampled by UVCS, to an enhanced temperature structure, that maintains its identity over a radial interval of $\approx 0.6 R_{\odot}$. This association, and the previous results from EIT and radio observations lead us to suggest that UVCS is observing the hot plasma of the CS region that extends from the low corona to the bottom part of the CME core. Hence, because the synoptic spectra we analyze do not include the FeXVIII line, we used the anticorrelated Si 499 and O $1032 \AA$ line intensities, their spatial coincidence with a LASCO-C2 bright feature and the earlier discussed facts from HXR, EIT, radio spectral and imaging data as evidence of a flare-CME-related CS site.

\section{Temperature and density derived from UV spectral lines}

Based on the UVCS data, we can demonstrate that a radial pattern in LASCO-C2 data which is situated between the planeof-sky-cut through the rising CME-flux rope and the previously identified probable position of the near-limb flare CS is filled with hot matter millions $\mathrm{K}$ above the surrounding corona. Here we explain some problems in deriving plasma temperatures from UVCS observations which are specific for the analyzed case.

As a result of our data analysis it turns out that we have a rare and extremely well adjusted data set crossing over several spectral ranges. The UVCS spectroscopic information is available just in spatial and time coincidence with one of the twisted 


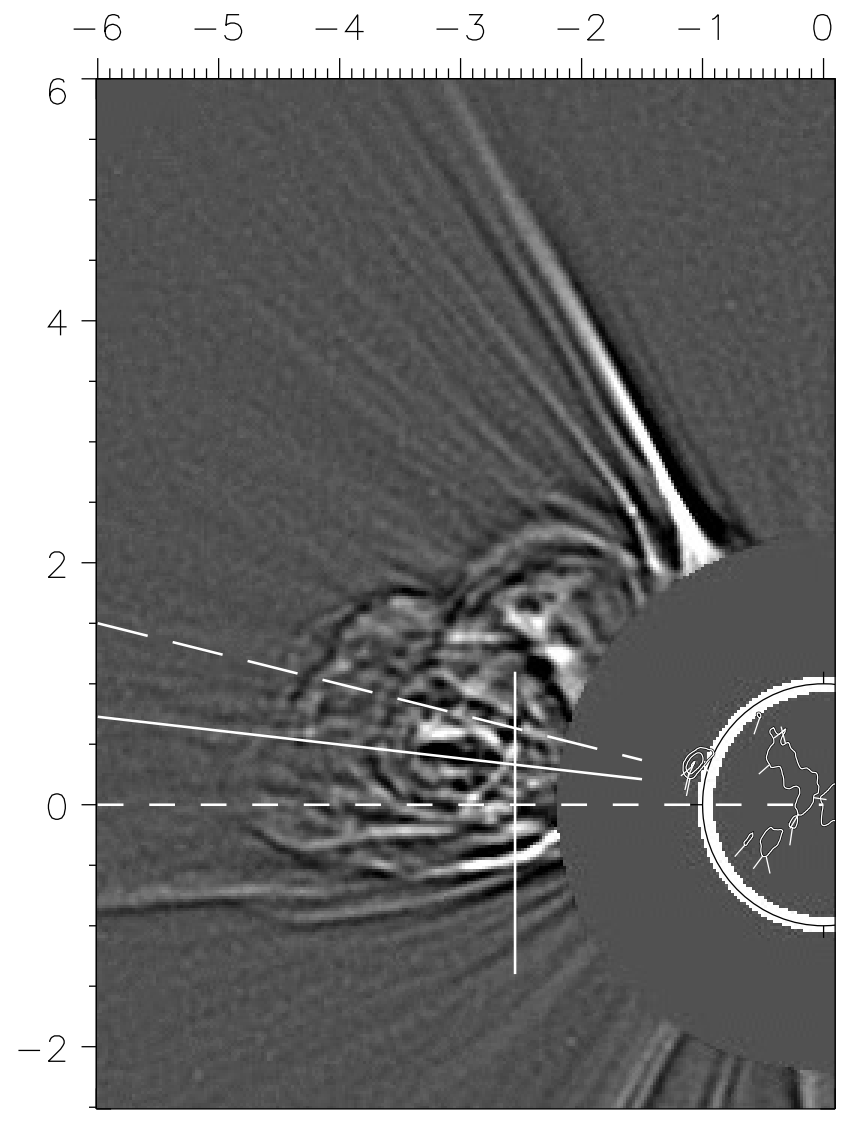

LASCO-C2 8:50

$\mathrm{NRH} 327 \mathrm{MHz} 8: 45-8: 55$

UVCS slit \& source pos. 8:46-9:13

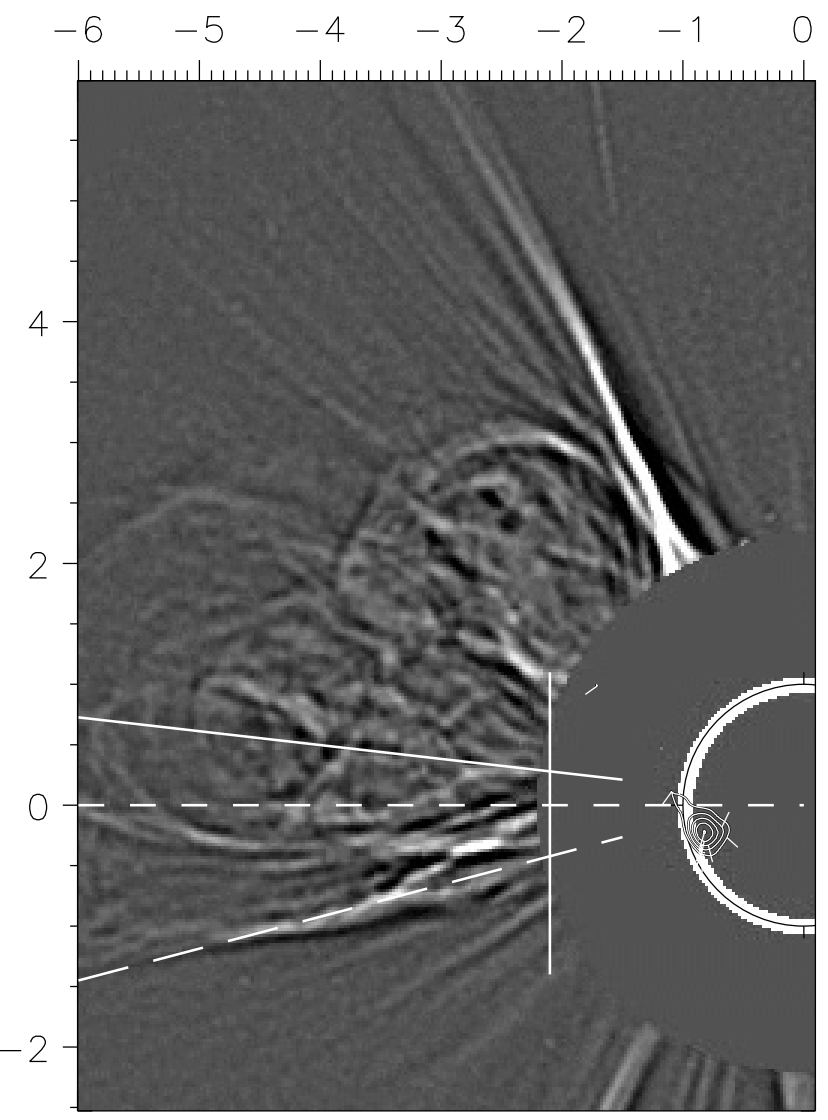

LASCO-C2 9:26

$\mathrm{NRH} 327 \mathrm{MHz}$ 9:11:38 type III 0 UVCS slit \& source pos. 9:13-9:30

Fig. 4. Combined SOHO white light coronograph images processed using a technique developed by Stenborg, and NRH $327 \mathrm{MHz}$ maps (white isolines at the limb, source PA indicated by the long dashed lines). The vertical line represents the UVCS spectrograph slit length and position. The short-dashed line denotes PA90 . Left panel: the NRH $327 \mathrm{MHz}$ map (8:45-8:55 UT) of long-term stable continuum emission (PA76 $)$ over the limb since NRH morning (8:27 UT; PA76 is also the position of the earlier SOHO-EIT loop (Fig. 2a) and the HXR sources between 07:31 and 07:45 UT; Karlický et al. 2004, and Table 1). Right panel: the type III burst at PA110 ${ }^{\circ}$ 09:11:38 UT in AIP spectral data (Table 1). In both panels, the solid radially oriented line at PA83 $3^{\circ}$ cuts through the radial structure under the dark core surrounded by white rims in the LASCO C2 image, and the SOHO-UVCS OVI minimum / SiXII maximum (see Fig. 5).

and radially elongated white light features formed in the corona during the CME evolution, that are radially aligned with the lower coronal radio and HXR sources observed during the earlier stages of the event.

In order to evaluate the temperature and density in the region where the high density white light feature crosses the UVCS spectral slit we need to take into account that while the SixII lines form, as usual for coronal lines, by collisional excitation, the H Ly- $\alpha$ and OVI coronal lines form also by resonant scattering of the disk radiation (the so called "radiative component"). The intensity of the radiative component, however, is a function of the speed of the emitting plasma, because of the Doppler Dimming effect (Beckers \& Chipman 1974) and may be completely eroded if the emitting plasma is moving outwards at a high enough speed.

The collisional line intensity (photons $\mathrm{cm}^{-2} \mathrm{~s}^{-1} \mathrm{sr}^{-1}$ ) may be written as:

$I_{\mathrm{el}}=A_{\mathrm{el}} \cdot \int_{\text {LOS }} \epsilon_{\text {line }} N_{\mathrm{e}}^{2} \mathrm{~d} x$ where $A_{\mathrm{el}}$ is the element abundance, $N_{\mathrm{e}}$ is the electron density (and we assumed $N_{\mathrm{e}}=N_{\mathrm{H}}$ ), $\epsilon_{\text {line }}$ is the line emissivity (photons $\left.\mathrm{cm}^{3} \mathrm{~s}^{-1}\right)$ that can be defined as $\epsilon_{\text {line }}=\frac{A_{\mathrm{el}}}{4 \pi} \frac{N_{\text {ion }}}{N_{\mathrm{el}}}\left(T_{\mathrm{e}}\right) C_{\mathrm{fj}}^{\mathrm{e}}\left(T_{\mathrm{e}}\right)$ where $A_{\mathrm{el}}$ is the absolute abundance of the element, $R_{\mathrm{el}}=\frac{N_{\mathrm{ion}}}{N_{\mathrm{el}}}$ is the ionization ratio, which is a function of temperature, $C_{\mathrm{fj}}^{e}\left(T_{\mathrm{e}}\right)$ is the coefficient of collisional excitation. The integral covers the region, along the line of sight (LOS), over which the line is emitted.

The ratio of intensities of two collisionally formed lines depends only on the electron temperature, once the element abundance is known. Hence, if we assume that OVI and SiXII form in a plasma with photospheric abundances, and we are able:

- to identify the contribution to the total line intensities originating from the CS region, and;

- to derive the Ovi collisional component,

we can easily infer its temperature. Figure 6 shows the emissivities of OVI $1032 \AA$ and SiXII $499 \AA$ and their ratio (left 


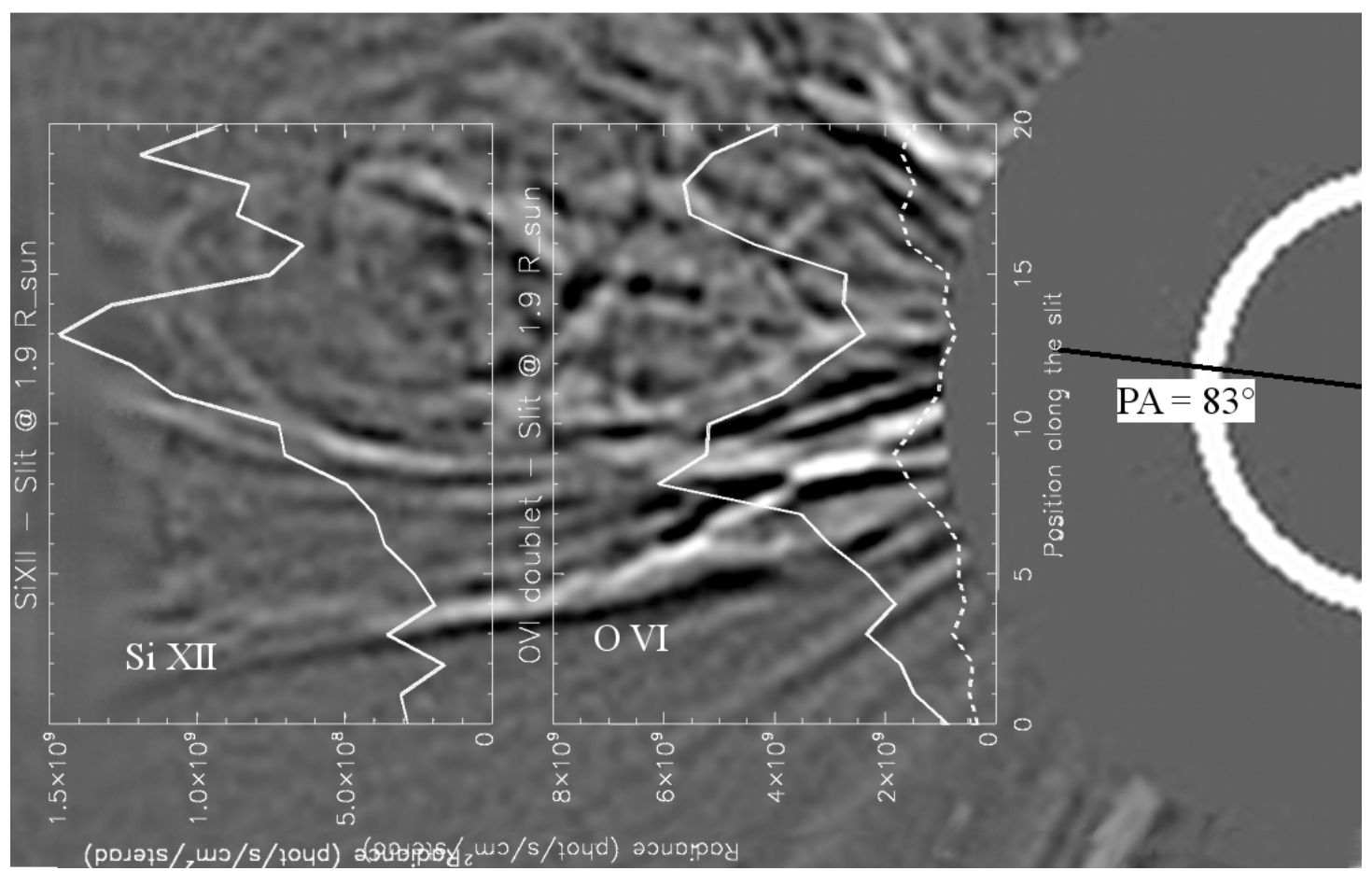

Fig. 5. Profiles of line intensity along the UVCS slit at 1.9 $R_{\odot}$ (9:30-9:43 UT) superposed on the LASCO-C2 contrast-enhanced image of 9:26 UT. The length of the $x$-axis corresponds to the length of the UVCS slit. The $x$-axis is normal to the equatorial radius to represent the actual slit position. The $y$-axis gives the total counts observed in the lines.

a)

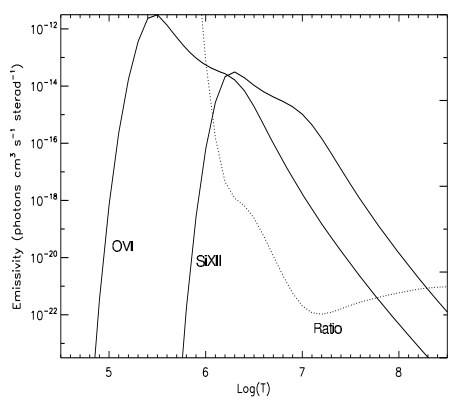

b)

Fig. 6. Estimate of temperature and density in a current sheet (CS) region embedded within the coronal plasma: a) emissivities of OVI $1032 \AA$ and SiXII $499 \AA$, and their ratio, from CHIANTI v5.0 with the photospheric abundances of Grevesse and Sauval (1998); b) along the line of sight (LOS) we suppose that the CS is surrounded by quiet coronal background plasma.

panel), as a function of the emission temperature. Data come from CHIANTI code, version 5.0 (photospheric abundances from Grevesse \& Sauval 1998) and show that the OVI 1032 line peaks at $\log T \approx 5.3-5.4$, while the SiXII 499 peaks at $\log T \approx 6.2-6.3$.

In order to apply the line ratio technique and derive the temperature in the CS region, we need first to separate the contribution of the quiet corona from the contribution arising in the CS region. We hypothesize that the hot plasma in the CS region is embedded within the quiet corona, as shown in the right panel of Fig. 6. Hence we need to subtract the quiet coronal background contribution from the line intensity before taking the ratio of their signals. This can be easily done for the SiXII line, whose quiet corona emission is given by the measured line intensity sideways of the CS region. However, the intensity of the OVI line

in the CS region is undistiguishable from that of the background corona. Hence we conclude that the OVI emission originating there is, if any, too weak to be identifiable and is completely blurred within the background emission. As a consequence, we may only assume, as an upper limit to the intensity of the OVI line in the CS-related parts of the image, a value given by the statistical uncertainty in the OVI intensity of the ambient corona.

We do not know whether lines in the CS form exclusively by collisional excitation (as it would occur if the CS region is pervaded by fast outflowing plasma) or whether we should consider also a radiatively excited component. This issue, however, is not relevant in our case, because the presence of a radiatively excited component would only decrease the value of the hypothesized collisional emission of the OVI line, which we already assumed to be an upper limit to its actual intensity in the CS.

The OVI/SiXII line intensity ratios, the infered temperatures and the electron densities corresponding to the minimum and maximum temperatures are summarized in Table 3. Higher temperatures would be obtained in case a radiative component contributes to the CS OVI line intensity. We notice, however, that the values we derived compare well with previous estimates of CS temperatures from the ratio of lines observed by UVCS and formed by collisional excitation only (e.g. Ciaravella \& Raymond 2008, derived values $\log T=6.6-6.9$ ).

Once the temperature is known, it is possible to derive the emission measure $\left(\mathrm{EM}, \mathrm{cm}^{-5}\right)$,

$E M=\int_{\mathrm{LOS}} N_{\mathrm{e}}^{2} \mathrm{~d} x$

and get an estimate of the electron density. This is only possible if a reliable estimate for the extension of the emitting plasma along the LOS is available. We assume the electron density to be roughly constant within the CS in question, so that we can approximate the integral with $N_{\mathrm{e}}^{2} \cdot \Delta x$, where $\Delta x$ is the thickness of 


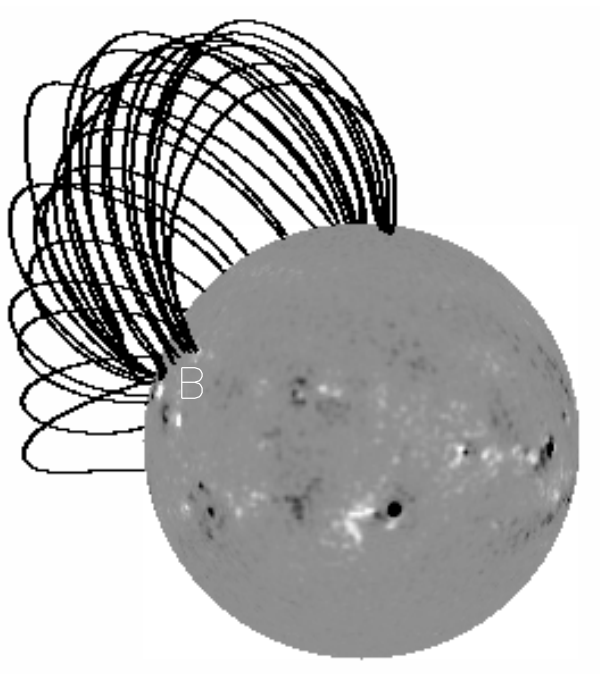

ARCADE SEEN FROM EARTH

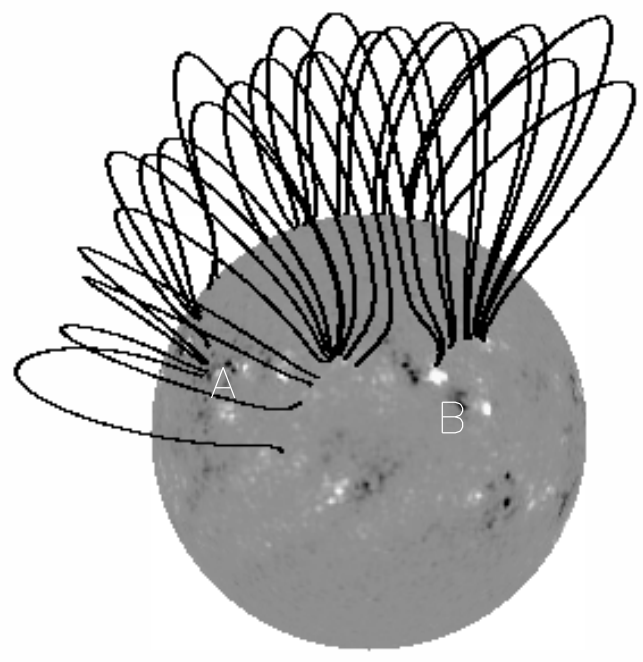

ARCADE SEEN FROM EAST a)

Fig. 7. Potential field (SOHO-MDI, 17 May 200206 UT) after Schrijver \& DeRosa (2003), closed field lines out of an a priori selected bounding box and inward field line tracing. Two perspectives are shown: a) as it appears on May 17, 2002; b) the same after rotating view a) 90 deg westward. A kinked arcade turns around the north-east limb from behind the sun. According to our view (Fig. 8b) the arcade between active regions A and B has a fundamental role in the formation of the $\mathrm{CME}$ and the flux rope embedded in it, as well as in triggering the flare in $\mathrm{B}$. the heated plasma layer across the CS. In order to facilitate the comparison with values derived by e.g. Vršnak et al. (2009), we assume $\Delta x \sim r$, where $r$ is the heliocentric distance, and adopt a reference thickness of $100 \mathrm{Mm}$ at $2.16 R_{\odot}$. At $1.5 R_{\odot}$, where our data are more reliable, we obtain, from the SiXII line intensity $N_{\mathrm{e}}=7.3 \ldots 10.9 \times 10^{7} \mathrm{~cm}^{-3}$, comparable with the electron density value given by Vršnak et al. (2009) at the same heliocentric height for a number of CS-like features imaged by the LASCO-C2 coronagraph.

\section{Discussion}

Based on the described observations we have been able to define a timeline of processes which should be summarized once again (compare Table 1):

- X-ray, radio data, and prominence observations show at the East limb a flare in AR 9957 (region B in Fig. 7b). Over the limb, HXR sources are reported associated with expanding coronal loops probably enclosing the eruptive flare filament.

- Radio- and X-ray flux over time are not simply related, the radio spectrum of the impulsive phase is quiet at meterand Dekameter waves. Decimeter waves show characteristic drifting pulsating structures (DPS) indicating dynamic processes in the stretched flare CSs underneath the rising filament.

- Radio data reveal between 07:56 and 08:00 UT:

- a faint narrowband pattern between 360 and $420 \mathrm{MHz}$;

- the diffuse onset of the CME continuum;

- a sudden reduction of the type III bandwidth;

- White light coronographs show the expanding CME. We identify stretched radial structures between the rising flux rope and the occulter.
- In one of the radial structures UVCS data reveal - after applying a specific line ratio method - an enhanced plasma temperature of $5 \times 10^{6} \mathrm{~K}$ well above the average corona.

- Radio data show after the fade-out of the CME continuum a single narrowband lane suggestive for a type II burst.

In order to reach a better understanding of the morphology of the large-scale structures we analyze, we need to understand how they are organized spatially. To this end we applied a potential field extrapolation of SOHO-MDI data (17 May 200206 UT) in spherical coordinates obtained thanks to the pfss-branch of solar software. It is important to note that we started field line tracing from large heights (see Schrijver \& DeRosa 2003, 171) to best reproduce structures determined by compact and strong surface sources approaching the LASCO-C2 occulter.

Figure 7a shows the sun in terrestrial observer's perspective. Overplotted are potential field lines rooted with one end in an a priori chosen starting box around active region 9957 (B). For simplicity, we have drawn the closed field lines in our region of interest, only. Looking from the Earth we see an arcade bridging over the NE-limb and apparently extending from region B up to the North pole. Figure $7 \mathrm{~b}$ is a view from the East limb (after rotating the sun from panel a) by $90 \mathrm{deg}$ westward $)^{6}$. We see now a giant kinked arcade visualized by field lines turning around the East and North limb and linking the behind-limb active region 9969/70 (NOAA SGD, A in Fig. 8b), and the region B. It is surprising how misleading the view a) of Fig. 7 can be, with respect to the $3 \mathrm{D}$ configuration we obtained.

Assuming that the (already kinked) arcade or a similar field structure further down gets de-stabilized starting behind the limb (e.g. in active region $\mathrm{A}$ of panel b) the different observed facts

\footnotetext{
${ }^{6}$ For the source of behind-sun information we refer to Schrijver \& DeRosa (2003).
} 
Table 3. Temperature and electron density estimate at various heliocentric heights (here corresponding to different observation times).

\begin{tabular}{cccc}
\hline \hline Height $\left(R_{\odot}\right)$ & OVI/SIXII & $\log (T)$ & $N_{\mathrm{e}}\left(\mathbf{c m}^{-3}\right)$ \\
\hline 2.1 & $0.06 \pm 0.04$ & $6.6 \pm 0.1$ & $3-5 \times 10^{6}$ \\
1.9 & $0.03 \pm 0.01$ & $6.6 \pm 0.1$ & $1 .-1.6 \times 10^{7}$ \\
1.7 & $0.02 \pm 0.01$ & $6.7 \pm 0.1$ & $2.2-3.2 \times 10^{7}$ \\
1.5 & $0.014 \pm 0.009$ & $6.8 \pm 0.1$ & $7.3-10.9 \times 10^{7}$ \\
\hline
\end{tabular}

become well compatible. Structures around B are always visible, structures near A must firstly reach the limb. This is one explanation of the impression of two CMEs in Fig. 2b. The features noticed in the radio spectra between 07:56 and 08:00 UT indicate the appearance of the CME over the limb and the corresponding disturbance and reconfiguration of the background field and the paths of energetic particle propagation (sudden type III bandwidth reduction).

The limb flare (Fig. 3) turns out to be the last energy release stage of the eruption before the CME lift-off in active region $\mathrm{B}$ near the E limb. The type III burst source at 9:11 UT (Fig. 4b) occurs where the potential field extrapolation consists of open field lines (not shown here). The burst source probably marks the outer rim of the CME-related disturbance projected on the disk. Due to the explained perspective effects the CME's leading edge in Fig. 7a is its last-detached part, and most of it moves away from earth behind the limb.

Figure 8a shows a LASCO-C3 difference image 12:42-12:18 UT. Dark arrows point to some white concave upward (Y-shaped) structures that trace the SE-NW inclination of the longitudinal axis of the rising flux-rope. In the foreground, the CME is diffuse, only one Y-shaped feature clearly showing up, while more can be seen at the NW section, although the (projected) CME morphology looks more complex in that region. White arrows in this difference image point to a bright arch situated under the large CME loops, and overlying a dark area ${ }^{7}$ near the coronograph's occulter. These bright/dark features in the difference frame might be formed by a rising pattern looking like an arch in projection. We argue that this features are the superposed lower ends of white light-visible structures (Y) which still connect the flux rope and the tops of post eruption loops.

Figure $8 \mathrm{~b}$ is a cartoon sketching the situation. Our scenario consists of a flux rope turning behind the limb and underneath supported by occasionally visible Y-shaped magnetoplasma structures. Klimchuk (1996) suggested - in agreement with soft X-ray and prominence observations - that there is no long plane CS existing under the rising destabilized arcade. He assumes that only a narrow fraction of the arcade field reconnects thus forming the flux rope with several singular CSs underneath. With this argument Klimchuk (1996) solves the energy budget problem which occurs if a huge plane CS reconnects. The repeated reconnection at different sites leads to repeated electron acceleration and to the repeated DPS patterns in decimeter radio spectra.

We have demonstrated that one of the radial coronographic structures corresponds to a hot plasma region in the wake of the rising CME (Hundhausen 1998; Saint-Hilaraire et al. 2009). We found that probably the flare CSs evolve into one or several of the larger-scale CME-wake CSs near the flare site. Our result coincides with predictions about the transition from a $2.5 \mathrm{D}$ to a 3D model of solar eruptive processes (summarized e.g. in Priest \& Forbes 1999; Somov 2000). It fits with earlier work on CSs in

\footnotetext{
7 No change in the time interval of the difference image!
}
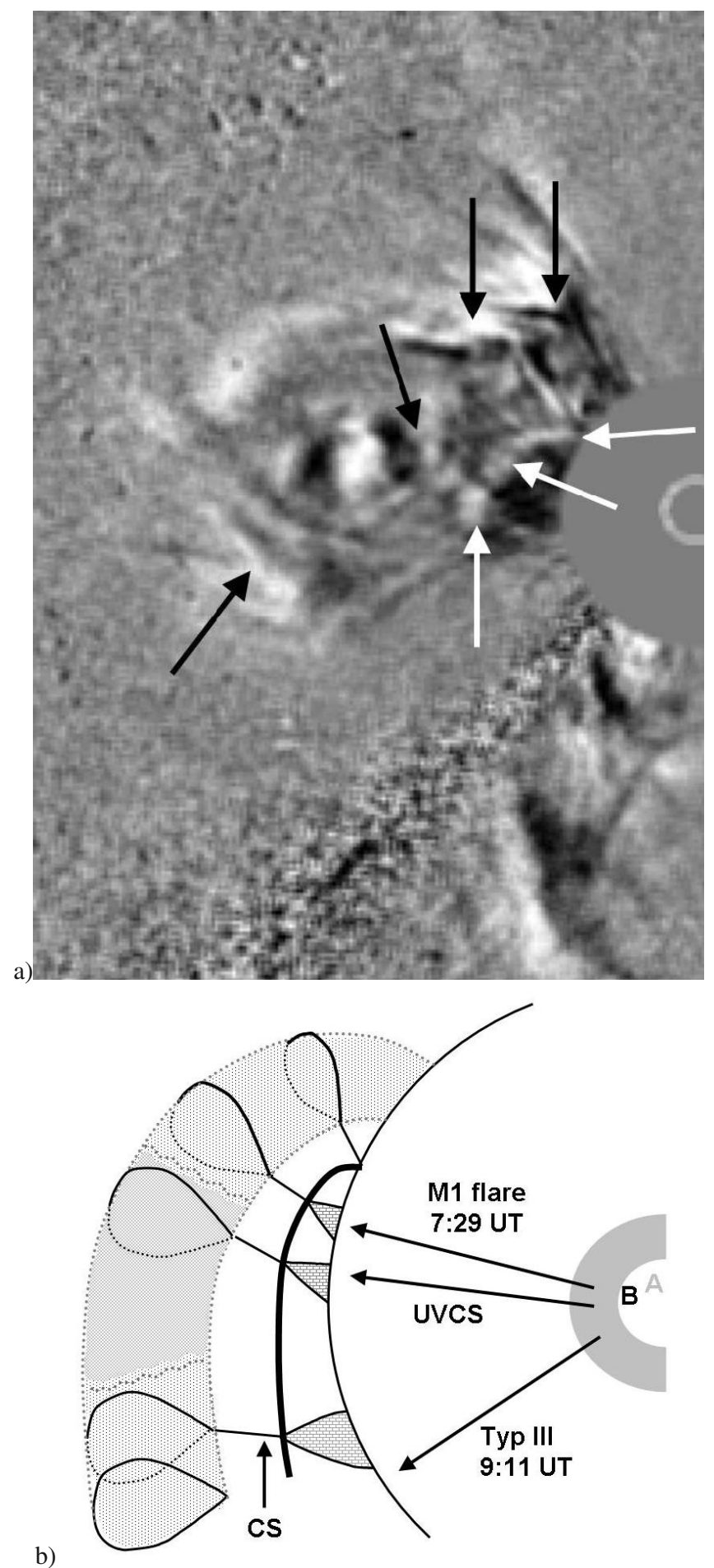

Fig. 8. The CME in the plane of sky. a) Part of SOHO-LASCO-C3 FOV, difference frame 12:42-12:18 UT. Dark arrows: some Y-patterns; white arrows: a white arch. b) Cartoon (not to scale) sketching authors viewpoint. The circle is the occulter at $5 R_{\odot}$. Arrows: point to high structures that project beyond the $\mathrm{C} 3$ occulter (see Fig. 4). The white arch of a) is drawn dark in b). Several CSs are stretched between that arch (connecting rising post-eruption loop tops) and the rising CME flux rope.

the wake of CMEs (e.g. Ko et al. 2003; Ciaravella \& Raymond 2008) that show regions of overheated coronal plasma aligned with white light-bright elongated plasma structures. Generally UVCS data refer to lower heights than those visible in LASCO $\mathrm{C} 2$, so we do not have a superposition (as we have here). 
The analyzed case is an interesting example illustrating the difficulties of the causality question between flares and CMEs. If our understanding of the observations is correct it is tempting to assume that the limb flare is driven by the already rising CME. The reconstruction of the global magnetic field structure yields a huge arcade extended from an active region on the backside of the sun to a near-E-limb region on the disk (regions A and $\mathrm{B}$ in Fig. 8b). A detailed analysis leads to the conclusion that the destabilization of a part of the corona and the formation of a CME probably starts behind the limb (near region A), and that the rising CME initiates later on the flare at position B. Our findings about the flare/CME relation in the given case are in agreement with the results of Zhang et al. (2001) and Gary \& Moore (2004) about the association between ongoing CMEs and flares.

From our interpretation of the data there follows another interesting conclusion with regard to the radio spectrum Fig. 3, the type II lane late in the event. Frequently the question is posed what drives the shock wave which eventually emits the radio radiation - the flare blast or the CME piston (e.g. Aurass 1997, but see also Warmuth \& Mann 2005). The type II burst definitely occurs much too late for a flare-blast driven shock from region $\mathrm{A}$ or from $\mathrm{B}$. Therefore we conclude that in this event, the coronal shock radio signature is driven by the expanding CME. Moreover, we can speculate ${ }^{8}$ because of the frequency range of the lane in the spectrum that this burst is driven by the flank of the CME because the head of the disturbance has already passed the corresponding height levels at that time.

\section{Conclusions}

In this paper we present the analysis of a comparatively simple event in several spectral ranges, and in a well suited position on the disk. From an analysis of radio and SOHO (UVCS, LASCO, EIT, MDI) data we derived independent indications favoring an interpretation in terms of one (or several radially elongated) current sheet(-s) formed early in the flare event and being later on observed above the $\mathrm{C} 2$ occulter in the wake of an ongoing CME. Some indications are indirect ones-in radio due to missing imaging, in white light due to the lack of information at heights covered by the occulter. So, we refer in radio on specific broadband drifting decimetric pulsations pointing on developing flare current sheets; and in white light on stable and radially elongated twisted features at sites where a current sheet is expected during the magnetic re-closure of the corona behind the $\mathrm{CME}$. The integrating element of the observations is the temporal and spatial sequence of effects during the flare/CME event considered together with the potential field extrapolation of photopheric field data in the expected magnetic footpoint range of the CME. UVCS observations have shown that one of the elongated features in white light images, radially linked with the previous limb flare site, is really associated with $\geq 5 \times 10^{6} \mathrm{~K}$ (heated) coronal matter. This means the coincidence of several indications of a current sheet in the expected timing, and in agreement with the coronal magnetic field structure confirms our view at least in the present case. To our knowledge this is one of the first published examples where it seems possible to argue for an interconnection between flare current sheets (usually underneath the rising active region filament) and one (of several) post-CMEcurrent sheets underneath the flux rope which rises as a part of the CME.
Acknowledgements. H.A. and G.P. thank the International Space Science Institute, Berne (ISSI) for the hospitality provided to the members of the team Role of Current sheets in Solar Eruptive Events where some of the ideas presented in this work have been discussed. We acknowledge the use of the Nançay Radio Heliograph, the SOHO instruments (Ultraviolet Coronal Spectrometer, Michelson Doppler Imager, Extreme Ultraviolet Telescope, and the Large Angle Spectroscopic Imager), the GOES satellite data, and the NOAA Solar Geophysical Data. SOHO is a project of international cooperation between ESA and NASA. We are grateful to M. Karlický (A.O. Ondřejov) for the access to the spectral and the $3 \mathrm{GHz}$ data. The LASCO-C2 images in Figs. 4 and 5 have been enhanced with a technique developed by Guillermo Stenborg. G.P. acknowledges support from ASI/INAF I/015/07/0. The critical remarks of the referee, Dr. K.-L. Klein, led to a substantial improvement of the presentation of the manuscript.

\section{References}

Anzer, U., \& Pneuman, G. W. 1982, Solar Phys. 79, 129

Aurass, H. 1997, in Coronal Physics from Radio and Space Observations, ed. G. Trottet, LNP, 483, 135

Aurass, H., \& Kliem, B. 1992, Solar Phys., 141, 371

Aurass, H., Vourlidas, A., Andrews, M. D., et al. 1999, ApJ, 511, 451

Bastian, T., Pick, M., Kerdraon, A., et al. ApJ, 558, 65

Bárta, M., Karlický, M., \& Büchner, J. 2009, Centr. Europ. Astrophys. Bull. 33, 299

Beckers, J. M., \& Chipman, E. 1974, Solar Phys. 34, 151

Benz, A. O. 1986, Solar Phys. 104, 99

Benz, A. O., Brajša, R., \& Magdalenić, J. 2007, Solar Phys. 270, 263

Biskamp, D. 2000, Magnetic Reconnection in Plasmas (Cambridge: Cambridge Univ. Press)

Ciaravella, A., \& Raymond, J. C. 2008, ApJ, 686, 1372

Crooker, N., Joselyn, J. A., \& Feynman, J. 1998, Coronal Mass Ejections, AGU Geophys. Monographs, 99

Dulk, G. A., McLean, D. J., \& Nelson, G. J. 1985, in Solar Radiophysics, ed. D. J. McLean, \& N. R. Labrum (Cambridge Univ. Press), 53

Gary, G. A., \& Moore, R. L. 2004, ApJ, 611, 545

Grevesse, N., \& Sauval, A. J. 1998, Space Sci. Rev., 85, 161

Hori, K. 1999, in Solar Physics with Radio Observations, ed. T. Bastian, N. Gopalswamy, \& K. Shibasaki, NRO Report, 479, 267

Hundhausen, A. 1998, in The Many Faces of the Sun (New York: Springer), 143 Karlický, M. 2004, A\&A, 417, 325

Karlický, M., Fárnik, F., \& Mészárosová, H. 2002, A\&A, 395, 677

Karlický, M., Fárnik, F., \& Krucker, S. 2004, A\&A, 419, 365

Khan, J. I., \& Aurass, H. 2006, A\&A, 457, 319

Kliem, B., Karlický, M., \& Benz, A. O. 2000, A\&A, 360, 715

Klimchuk, J. A. 1996, in Magnetic Reconnection in the Solar Atmosphere, ASP Conf. Ser. 111, 319

Ko, Y.-K., Raymond, J. C., Lin, J., et al. 2003, ApJ, 594, 1068

Lin, J., Ko, Y.-K., Raymond, J. C., et al., ApJ, 622, 1251

Mann, G., Jansen, F., MacDowall, R. J., Kaiser, M. L., \& Stone, R. G. 1999, A\&A, 348, 614

Martens, P. C. H., \& Kuin, N. P. M. 1989, Solar Phys. 122, 263

Masuda, S., Kosugi, T., Hara, H., Tsuneta, S., \& Ogawara, Y. 1994, Nature, 371, 495

Noci, G., Kohl, J. L., \& Withbroe, G. L. 1987, ApJ, 315, 706

Newkirk, G. A. 1961, ApJ, 133, 983

Poletto, G., Suess, S. T., Bemporad, A., et al. 2004, ApJ, 613, L173

Priest, E., \& Forbes, T. 1999, Magnetic Reconnection-MHD Theory and Applications (Cambridge Univ. Press)

Saint-Hilaire, P., Krucker, S., \& Lin, R. P. 2009, ApJ, 697, 245

Schrijver, C. J., \& DeRosa, M. L., Solar Phys. 2003, 212, 165

Somov, B. V. 2000 Cosmic Plasma Physics (Dordrecht: Kluwer), 574

Śvestka, Z. F., Poletto, G., Fontenla, J., et al. in The Many Faces of the Sun, ed. K. T. Strong, J. L. R. Saba, B. M. Haisch, \& J. T. Schmelz (New York: Springer), 409

Tsuneta, S., Hara, H., Shimizu, T., et al. Proc. Astron, Soc. Jap. 44, L63

Vršnak, B., Poletto, G., Vujić, E., et al. 2009, A\&A, 499, 905

Warmuth, A., \& Mann, G. 2005, A\&A, 435, 1123

Webb, D. F., Burkepile, J., Forbes, T. G., et al. 2003, JGR, 108, A12, 1440

Zhang, J., Dere, K. P., Howard, R. A. 2004, et al. ApJ, 604, 420, 2004

\footnotetext{
${ }^{8}$ No imaging data available in this frequency range on that day.
} 